\title{
2.5 years on: what are the effects of a 'frequent attenders' service in the Emergency Department?
}

\author{
Soraia Sousa, ${ }^{1}$ Tracey Hilder, ${ }^{1}$ Christopher Burdess, ${ }^{2}$ Philippa Bolton ${ }^{1}$
}

BJPsych Bulletin (2019) 43, 112-116, doi:10.1192/bjb.2019.8

${ }^{1}$ Lanchester Road Hospital, Durham, UK; ${ }^{2}$ University Hospital North Durham, Durham, UK

Correspondence to Dr Soraia Sousa (soraia.sousa@gmail.com)

First received 26 Feb 2018, final revision 20 Oct 2018, accepted 4 Jan 2019

(c) The Authors 2019. This is an Open Access article, distributed under the terms of the Creative Commons Attribution-NonCommercialNoDerivatives licence (http:// creativecommons.org/licenses/by-ncnd/4.0/), which permits noncommercial re-use, distribution, and reproduction in any medium, provided the original work is unaltered and is properly cited. The written permission of Cambridge University Press must be obtained for commercial re-use or in order to create a derivative work.
This study focused on an evaluation over 2.5 years to establish if a frequent attenders' service in an Emergency Department (ED) impacted on the overall number of patients attending as well as the numbers of their attendances. For this, three patient lists from April-September 2015 and three lists from a matched period in 2017 were randomly selected and the two samples compared. Results showed both a reduction in the number of total patients identified as frequent attenders as well as a reduction on the number of attendances to ED. The study suggests that the implementation of a frequent attenders' service is associated with benefits, not only for the individual attendances per patient, but also in an overall reduction of the number patients classed as frequent attenders.

Declaration of interest The authors have no conflict of interest to declare regarding this manuscript.

Keywords Frequent attenders; emergency department; high attenders; accident and emergency.
Patients who frequently attend the Emergency Department (ED) have been characterized as a heterogeneous group, ${ }^{1,2}$ often with complex healthcare needs who are at least as sick as non-frequent attenders ${ }^{2,3}$ and have been associated with a higher expected mortality rate. ${ }^{1,4}$ A recent national Commission for Quality and Innovation (CQUIN) aimed to improve the way services manage these patients. This was based on the Cambridge study ${ }^{5}$ which highlighted that there should be services designed to integrate physical and psychological needs of these patients at the ED interface. In the current CQUIN, a cohort of patients who attend frequently is identified, and the pre and post intervention attendances across the cohort are compared with a $20 \%$ reduction in attendances required to achieve the target.

However, in the UK, the longer term effect of a frequent attenders' (FA) service has not been evaluated in terms of whether such a service has an impact on the overall number of patients attending ED who are classed as 'frequent attenders'.

In Durham and Darlington a FA's service has been running since 2014 following the implementation of a local CQUIN with County Durham and Darlington National Health Service (NHS) Foundation Trust in 2014/2015 which aimed to reduce attendances in those attending the Emergency Department (ED) 20 times a year or more. Tees, Esk and Wear Valleys (TEWV) NHS Foundation Trust was also involved in providing mental health support via the Psychiatric Liaison team, and the North East Ambulance service were the third partner in this project. This CQUIN was commissioned in response to guidelines from the Royal College of Emergency Medicine (August 2014). ${ }^{6}$ Due to the relatively small numbers of patients attending over 20 times a year the service saw all patients attending 15 times a year or more.

The initial local CQUIN demonstrated a $46 \%$ reduction in attendances in the cohort over a 12 month period compared to the previous 12 months and every patient demonstrated a reduction in attendance. Similar outcomes have been showed in other studies. ${ }^{7,8}$

This service has been in place 2.5 years at the time of this study and an evaluation of its impact over this time period was conducted. This evaluation aims to establish if a FA's service impacts on the overall number of patients attending frequently as well as the overall frequency of their attendances to the ED.

\section{Methods}

The Frequent Attenders Service has been set up between the ED of the University Hospital of North Durham in Durham and the Liaison Psychiatry team. Patients are identified as frequent attenders by the ED if they attend 15 times a year or more. Each month a list is produced from the ED of all these patients. The list will have on it any patient who has attended the ED department 15 times or more in the previous year with no other exclusion criteria applied. The 


\begin{tabular}{|c|c|c|c|c|c|c|c|c|c|c|}
\hline Month (List) & 2015 & Mean & SD & 2017 & Mean & SD & $95 \% \mathrm{Cl}$ for Mean & $\mathrm{T}$ & Df & Sig. (2-tailed) \\
\hline 1 & 41 & \multirow[t]{3}{*}{43.33} & \multirow[t]{3}{*}{4.04} & 13 & \multirow[t]{3}{*}{16.67} & \multirow[t]{3}{*}{5.51} & \multirow[t]{3}{*}{$15.71,37.61$} & \multirow[t]{3}{*}{6.76} & \multirow[t]{3}{*}{4} & \multirow[t]{3}{*}{0.0025} \\
\hline 2 & 41 & & & 23 & & & & & & \\
\hline 3 & 48 & & & 14 & & & & & & \\
\hline
\end{tabular}

patients on the list are discussed at a monthly MultiDisciplinary Team (MDT) meeting with ED, Liaison Psychiatry and the North East Ambulance Service (NEAS). The number of patients in the list for each month is dynamic as each month there will be new patients who meet criteria, some patients will be discharged as they have reduced their attendances and others who will continue on from the preceding month's list.

For some patients already involved in services, liaison with their established care providers is undertaken as often those providers are not aware of the ED attendances and are able to address the situation with the patient effectively within current care provision. For other patients a letter is sent offering them an appointment with the Liaison Psychiatry team, sometimes in collaboration with a different team - e.g. a joint consult with the respiratory nurses and Liaison Psychiatry. Plans of care are put in place for all patients with contributions from the MDT and the patient, if the patient chooses to engage. If the patient does not engage, a plan is put in place anyway if there are sufficient concerns about risk e.g. a plan may ensure that a patient with health anxiety does not receive iatrogenic harm from unnecessary tests. Education is also done with ED clinicians to look at approaches to talking to patients who frequently attend and potential management strategies. Two liaison clinicians (consultant psychiatrist and advanced nurse practitioner) coordinate the service and contribute 1 session a week between them on average.

This evaluation was conducted by randomly selecting three patient lists between April-September of the year 2015 and comparing this data to three randomly selected patient lists from a similar period in 2017. This was done to ensure that the result was representative and not skewed by a single particularly good or bad month. Therefore, the total number of patients for each list was obtained and their average number calculated.

Other data collected to compare the two cohorts was: age, gender, and most common clinical presentation. One list from each year only was used for this extra information. During the evaluation we followed up the patients who had attended in 2015 to analyse how many were still attending frequently in 2017. During this we noted how many had died and what their original reason for attending was. This mortality data is also presented in the results.

The data was collated and analysed using Microsoft Excel 2010. Consent from patients and ethical approval was not sought given that the study was a service evaluation of an established service and no patient identifiable information is presented.

\section{Results}

\section{Analysis of the average number of patients attending 15 times a year or more}

Considering the period between April-September 2015, an average of 43 patients were attending ED 15 times a year or more, whereas this number reduced to an average of 17 patients in 2017 as shown in Table 1 . This equates to a reduction of $60 \%$ in the average number of patients attending 15 times a year or more over the period of 2.5 years.

An independent sample t-test was conducted to compare the means of the samples for 2015 and 2017. There was a statistically significant difference between the means of the number of patients per month in 2015 when compared to 2017 (t (4) $=6.76, P=0.0025$ ).

\section{Analysis of the average number of total attendances amongst the frequent attenders over preceding 12 months in 2015 compared to 2017}

As can be seen in Table 2, the average number of total attendances over preceding 12 months by patients attending 15 or more times a year reduced from approximately 833 to 437 which is 396 fewer attendances. This amounts to a $48 \%$ reduction on the total number of attendances across the 2.5 years of the study. This difference was shown to be statistically significant $(t(4)=6.38, P=0.0031)$.

\section{Descriptive analysis of age, gender and clinical presentation to ED}

As stated in the methods section, for this part of the analysis only one random list of each year was considered. For both

\begin{tabular}{|c|c|c|c|c|c|c|c|c|c|c|}
\hline Month (List) & 2015 & Mean & SD & 2017 & Mean & SD & $95 \% \mathrm{Cl}$ for Mean & $\mathrm{T}$ & $\mathrm{df}$ & Sig. (2-tailed) \\
\hline 1 & 731 & \multirow[t]{3}{*}{833.33} & \multirow[t]{3}{*}{89.97} & 393 & \multirow[t]{3}{*}{436.67} & \multirow[t]{3}{*}{59.16} & \multirow[t]{3}{*}{$224.04,569.28$} & \multirow[t]{3}{*}{6.38} & \multirow[t]{3}{*}{4} & \multirow[t]{3}{*}{0.0031} \\
\hline 2 & 869 & & & 504 & & & & & & \\
\hline 3 & 900 & & & 413 & & & & & & \\
\hline
\end{tabular}


years, each list considered showed a small majority of female patients (55\% in 2015 and 57\% in 2017). The median age of patients in 2015 was lower than in 2017 (36v. 43 years old) (IQR 28.5-53 v. IQR 33-65).

Both samples also showed a similar distribution for clinical presentation to ED. Personality disorders and alcohol and substance misuse lead the most common clinical presentations followed by pain, undiagnosed dementia and chest problems (most commonly patients with respiratory problems) as it can be observed in Fig. 1. The Figure also shows the general reduction in the number of patients attending ED. However, this reduction is not the same across the different clinical presentations with the categories of undiagnosed dementia, children under 18, complex physical health needs and other remaining unchanged from 2015 to 2017. On the other hand, a reduction is seen in patients attending with alcohol and drug misuse problems, respiratory problems, pain and borderline personality disorder.

\section{Analysis of outcomes for patients across the $\mathbf{2 . 5}$ year period of the study}

It was also observed that all the patients who had been identified as frequent attenders in 2015 reduced their number of attendances to less than 10 per year by 2017 (some were not attending at all) and consequently they were no longer on the FA list, with the exception of a single patient (Patient A). Patient A is the only patient to be present in the FA's list in 2015 and 2017 and has also the highest number of attendances per year of both groups.

Of 13 elderly patients who presented as FA throughout the 2.5 period analysed, 11 had a previously undiagnosed dementia and almost all of those eventually required residential care.

It was noted that of the patients who attended in 2015, 9 had died 2.5 years later. Of these 2 were elderly patients who died of natural causes, one was a patient with a terminal illness and 5 were patients with substance abuse, 4 of which were alcohol related. In fact, when examined more closely, of the 8 original patients with alcohol presentations in 2015,
4 died within 18 months of identification as being a frequent attender. Also, of note, none of the patients in 2015 who presented with a primary presentation of self-harm or suicide attempts had died in the following 2.5 years.

\section{Discussion}

The implementation of a MDT approach to reduce attendance to ED services by frequent attenders has been suggested as effective by a number of studies $^{7,8}$ and has therefore been recommended by the College of Emergency Medicine. However, a significant number of studies regarding this have been done outside of the UK which has implications on how much information can be extrapolated towards the UK population given significant differences in the national health care systems.

This study observed that since the implementation of the FA's Service, a marked reduction of the number of patients classed as 'frequent attenders' occurred from an average of 43 patients falling into this category each month in 2015 compared to an average of 17 patients in 2017. The average number of attendances of these patients for the preceding 12 months also reduced from approximately 833 to 437. Both reductions were statistically significant.

The reduction in the number of patients on the monthly frequent attenders list from implementation of the service was unexpected. A number of factors might have contributed to this result. As it was presented in the results, with exception of patient A, all patients clustered as frequent attenders in 2015 reduced their number of attendances and did not qualify for this category by 2017, which means that the 2017 patients' list (with the exception of patient A) was made of new patients. This means that there isn't a cumulative effect by which the list expands year after year as new patients are added. This has also been discussed by other studies. In two studies ${ }^{2,8}$ it has been demonstrated that some patients will qualify as FA during a period of crisis which is not a situation that repeats year after year. Therefore, each cohort tends to have a new set of patients while others are removed from the list.
Fig. 1 Clinical presentations to ED in absolute numbers. A/S misuse, alcohol and/or substance misuse; BPD, borderline personality disorder; HA/MUPS, health anxiety/medically unexplained psychiatric symptoms; $\mathrm{PH}$ physical health.

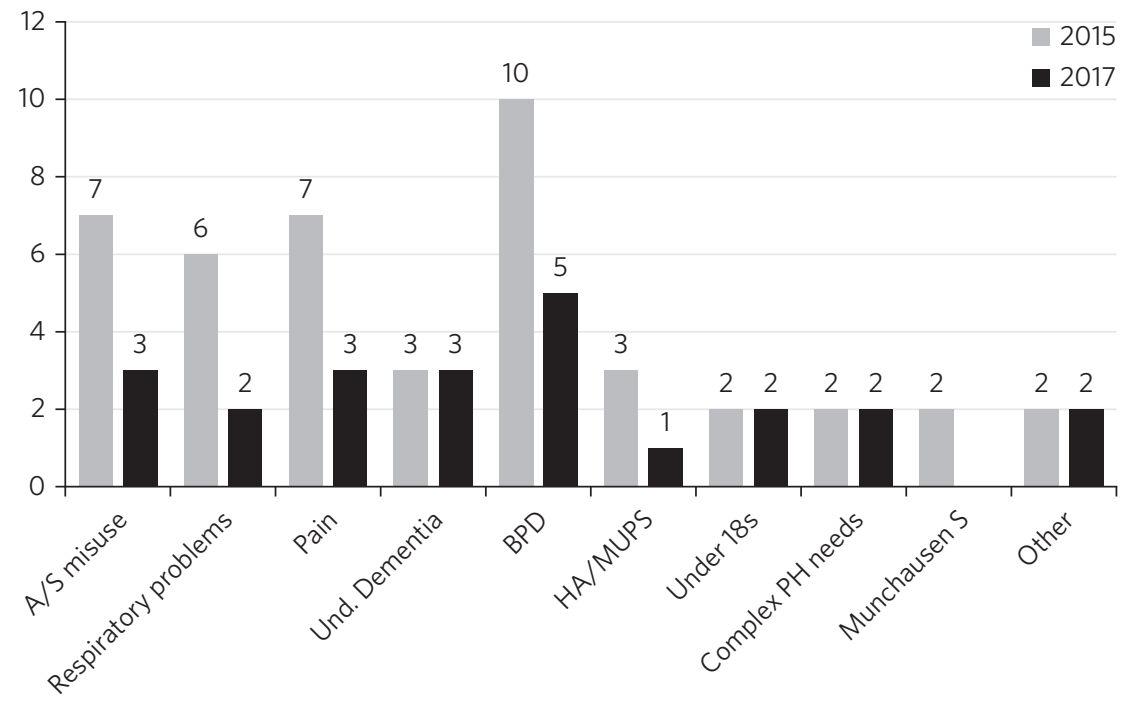


Another potentially contributing factor to this result is the educational component of the project in which ED clinicians are encouraged to identify patients earlier and utilise more effective management strategies thereby reducing the potential of the patients to become frequent attenders.

The reason why early identification of these patients contributes to reduction in their attendance could be related to early addressing of potential conditioned responses that some patients develop by going to ED. For example, in acute exacerbations of chronic pain, the patient learns that ED will provide IV analgesics which will be more effective than oral therapy due to associated immediate euphoria and deep relaxation rather than just the analgesic effect. Hence during acute exacerbations of chronic pain coming to ED becomes the learned response. There is also an association of hospital in general as a place of safety and comfort which has been referred to as the "brick mother" by psychotherapist Henri Rey. Therefore, the patient learns that his fear, which will greatly impact on pain, will be more favourably managed in hospital than at home and this gives rise to a further conditioned response. By acting early, this response can be addressed before it becomes deeply entrenched and therefore patients can be better managed in the community e.g. in conjunction with support from the pain team. Overall practice has changed as well, with fewer clinicians prescribing IV analgesics for acute exacerbations of chronic pain and there have been fewer patients attending with acute exacerbations of chronic pain as can be seen in Fig. 1.

Another way behavioural reinforcers have been addressed is by taking a positive risk approach to physical health presentations, in particular in patients with health anxiety. This has been done by minimizing the number of investigations carried out in patients who have been appropriately investigated and who do not present with new symptoms. This has benefits for the system as it reduces the cost of the emergency episode but also for the patient as it reduces iatrogenic harm by repeated exposure to unnecessary procedures and harmful ionising radiations. A consistent approach to this also helps stop the chain of reinforcement.

It has been noted that for some patients, simply being sent a letter informing them that they are a frequent attender and offering help to address this seems enough to stop them attending. For others it is about getting the relevant community services to work better for the patient - e.g. often mental health services will not be aware that a patient is attending $\mathrm{ED}$ frequently and this can be addressed through crisis plans. Working with the community respiratory team has been highly effective in helping patients who repeatedly present with respiratory concerns, or referring patients to the pain clinic to come up with innovative community ideas for managing acute exacerbations of chronic pain have helped. These interventions appear to be the most likely explanation to the difference in the reduction of different clinical presentations seen in Fig. 1, with the highest reductions in pain, respiratory problems and borderline personality disorder presentations.

In this study, female gender was predominant, whereas a number of studies ${ }^{3,9-11}$ have showed higher prevalence of males in groups of frequent attenders. The mean age of patients in the second cohort was 7 years younger and generally this study presented lower mean ages than other studies $^{9,11}$. Some studies ${ }^{1,10}$ have also suggested that the age distribution is bi-modal with peak ages in younger patients ( $<40$ years old) and old age ( $>65$ years old).

Two unexpected groups emerged that warrant further research: firstly, older people who attended ED frequently often had undiagnosed dementia and potentially older people frequently attending ED could be a red flag for considering this. Secondly, substance misuse patients in this study have a very poor prognosis. Half of the 2015 patients identified as FA with substance misuse problems have now died. This has serious implications for how these patients are cared for - they often do not engage in usual substance misuse services and this warrants further consideration as to what services models are needed to best support these high risk patients.

Regarding the costs of frequent attenders to $\mathrm{ED}$ : Considering the distribution of common presentations to ED in our sample, it is likely that basic investigations such as blood analysis or an x-ray are done which means that the cost per presentation will be around $£ 127 .{ }^{12}$ If a more specialized investigation such as a computerized tomography (CT) or ultrasound scan is done, the cost further increases to around $£ 207 .{ }^{12}$ This calculation does not take into consideration inpatient admissions (average cost $£ 400$ per night), or the use of ambulances (average cost of $£ 254$ per attendance). ${ }^{13}$ More than this, though, are the costs related to excess mortality and morbidity in this group of patients, whose underlying conditions are not being optimally managed, potential iatrogenic harm from unhelpful tests, investigations and treatments, poor patient and clinician experience and the disproportionate amount of time these patients take up in the ED due to their complexity. Of note, in the original CQUIN of patients attending over 20 times a year, there was a reduction of 170 inpatient admissions to the acute trust in the 12 months prior to the intervention compared to the 12 months post intervention.

There are a number of limitations to this evaluation and results should not be interpreted too broadly. The sample was collected from the area covered by County Durham and Darlington NHS Foundation Trust hospitals which covers a population with its unique characteristics that differ from other areas. Moreover, the sample for the study was small and therefore limits generalization into the general population. Another important issue, as mentioned above is the availability of other services which conditions the type of patients presenting to ED. That said, most literature reviewed concentrated on the effects of service implementation and therefore focused on assessing a single cohort of patients pre and post intervention and analysis of those individual's reduction of attendance whereas this service evaluation was able to show broader benefits in terms of actually reducing the number of frequent attenders rather than only the number of attendances per patient.

In conclusion: a frequent attenders' service can be effective in reducing the overall number of patients attending ED 15 times a year of more. This has led to the service being able to see patients attending 10 times a year or more. The most effective elements of the service are hypothesised as being: the psychoeducation of clinicians; 
working with patients and other relevant agencies to help them manage their condition in the community more effectively; identifying and breaking the cycle of the conditioned response these patients show to emergency services and the liaison between different services.

\section{Case study}

Ms D had a long history of COPD exacerbated by anxiety and previously attended ED over 40 times a year. Following a period of brief intervention with the frequent attenders' clinic which involved: joint work with the respiratory community team; a plan with the ambulance service to differentiate between an anxiety episode and COPD; a management plan for ED; the patient becoming involved with a community support group with the respiratory team; support with the local housing and social support team. Since this intervention was put in place Ms D has attended ED only once a year in the last 2 years (both appropriately) and has had one additional ambulance call where the crew managed the presentation at home.

\section{About the authors}

Dr Soraia Sousa is a Trust Doctor in Psychiatry in the Tees, Esk and Wear Valleys NHS Foundation Trust, UK. Mrs Tracey Hilder is an Advanced Nurse Practitioner for Tees, Esk and Wear Valleys NHS Foundation Trust working in the Liaison Psychiatry team at University Hospital of North Durham, UK. Dr Christopher Burdess is an Accident and Emergency Consultant for County Durham and Darlington NHS Foundation Trust working at the University Hospital of North Durham, UK. Dr Philippa Bolton is a Liaison Consultant Psychiatrist for Tees, Esk and Wear Valleys NHS Foundation Trust working in the Liaison Psychiatry team at University Hospital North Durham, UK.

\section{References}

1 LaCalle E, Rabin E. Frequent users of emergency departments: the myths, the data, and the policy implications. Ann Emerg Med 2010; 56 (1): 42-8. Available from: doi:10.1016/j.annemergmed.2010.01.032.

2 Ruger JP, Richter CJ, Spitznagel EL, Lewis LM. Analysis of costs, length of stay, and utilization of emergency department services by frequent users: implications for health policy. Acad Emerg Med 2004; 11(12): 1311-7. Available from: doi:10.1197/j.aem.2004.07.008.
3 Moore L, Deehan A, Seed P, Jones R. Characteristics of frequent attenders in an emergency department: analysis of 1-year attendance data. Emerg Med J 2009; 26: 263-7. Available from: doi:10.1136/emj.2008/ 059428.

4 Hansagi H, Olsson M, Sjoberg S, Tomson Y, Goransson S. Frequent use of the hospital emergency is indicative of high use of other health care services. Ann Emerg Med 2001; 37: 561-7. Available from: doi:10.1067/ mem.2001.111762.

5 FACE Steering Group. Frequent Attender, Care Enhanced (FACE) Team. Cambridgeshire and Peterborough Clinical Commissioning Group. 2013.

6 The College of Emergency Medicine. Frequent Attenders in the Emergency Department: Best Practice Guideline. 2014. Available from: https://www.rcem.ac.uk/docs/College\%20Guidelines/5x.\%20Frequent \%20Attenders\%20in\%20the\%20Emergency\%20Department(August $\% 20$ 2014).pdf (accessed 1 November 2017).

7 Soril LJJ, Leggett LE, Lorenzetti DL, Noseworthy TW, Clement FM. Reducing frequent visits to the emergency department. A systematic review of interventions. PLOS ONE 2015; 10(4): e0123660. Available from: doi:10.1371/journal.pone.0123660.

8 Skinner J, Carter L, Haxton C. Case management of patients who frequently present to a Scottish emergency department. Emerg Med 2009; 26: 103-5. Available from: doi:10.1136/emj.2008.063081.

9 Byrne M, Murphy AW, Plunkett PK, McGee HM, Murray A, Bury G. Frequent attenders to an emergency department: a study of primary health care use, medical profile, and psychosocial characteristics. Ann Emerg Med 2003; 41: 309-18. Available from: doi:10.1067/ mem.2003.68.

10 Chan JSE, Tin AS, Chow WL, Tiah L, Tiru M, Lee CE. Frequent attenders at the emergency department: an analysis of characteristics and utilisation trends. Proc Singapore Healthcare 2017; 27(1): 12-9. Available from: doi:10.1177/2010105817715271.

11 Phillips GA, Brophy DS, Weiland TJ, Chenhall AJ, Dent AW. The effect of multidisciplinary case management on selected outcomes for frequent attenders at an emergency department. Med J Aust 2006; 184(12): 602-6. Available from: https://www.researchgate.net/publication/6982142_The_effect_of_multidisiplinary_case_management_on_ selected_outcomes_for_frequent_attenders_at_an_emergency_department (accessed 1 November 2017).

12 NHS Improvement. National Tariff Payment System 2017/2018 and 2018/ 2019. Available from: https://improvement.nhs.uk/resources/nationaltariff-1719/\#h2-annexes (accessed 16 January 2018).

13 Morse A. NHS Ambulance Services. National Audit Office. 2017 Available from: https://www.nao.org.uk/wp-content/uploads/2017/ 01/NHS-Ambulance-Services.pdf (accessed 16 January 2018). 